\title{
Mapuchea pinoi sp. nov. (Hemiptera: Membracoidea: Myerslopiidae): especie fósil de un lecho de turba de Monteverde, región de Los Lagos, Chile
}

\author{
Mapuchea pinoi sp. nov. (Hemiptera: Membracoidea: Myerslopiidae): \\ a fossil species from a peat bead of Monteverde, Los Lagos region, Chile
}

Juan F. Campodonico ${ }^{1}$, Eduardo I. Faúndez ${ }^{2,4}$ \& Allan C. Ashworth ${ }^{3}$

\section{Resumen}

Se describe Mapuchea pinoi sp. nov. (Myerslopiidae) a partir de material fósil de Monte Verde, a orillas de la cala de Chinchihuapi, región de Los Lagos, Chile, en base a un tegmen izquierdo, con una data de 13.429 años AP. A pesar de ser una especie descrita en base a material fósil, se cree que esta especie podría no encontrarse extinta.

\section{Palabras clave:}

Myerslopiidae, Mapuchea, nueva especie, Chile, Monte Verde, fósil.

\begin{abstract}
A fossil myerslopiid, Mapuchea pinoi sp. nov., is described from Monte Verde, Los Lagos Region, Chile. The new species is described on basis of a left tegmen, of 13,429 yr. BP. Although it is a fossil species it is possible that this taxon is not extinct.
\end{abstract}

\section{Key words:}

Myerslopiidae, Mapuchea, new species, Chile, Monte Verde, fossil.

\section{INTRODUCCIÓN}

Myerslopiidae Evans, 1957 es una pequeña familia de hemípteros, con una vida ligada al horizonte orgánico del suelo y que aparentemente se alimentan a partir de raíces y tallos de angiospermas (Rakitov, 2015). La familia se encuentra conformada por los géneros
Myerslopia Evans, 1947 y Pemmation Hamilton, 1999, de Nueva Zelanda, y Mapuchea Szwedo, 2004, de Chile; a lo que se le suma el género fósil Ovojassus Hamilton, 1990 de la formación Santana (Brasil), cretácico inferior, aptiense (Szwedo, 2004b).

El género Mapuchea Szwedo, 2004 se encuentra actualmente representado por las especies Mapuchea burckhardti Szwedo, 2004 (región de Coquimbo en el Parque Nacional Fray Jorge); Mapuchea hamiltoni Szwedo, 2004 (región de la Araucanía) y Mapuchea chilensis (Nielson, 1996) (Regiones de la Araucanía, Los Ríos y Los Lagos) (Nielson, 1996; Szwedo, 2004a; 2004b; Rakitov, 2015).

La presente contribución tiene por objeto describir una nueva especie de Mapuchea a partir del fósil de un tegmen izquierdo, encontrado en el sitio arqueológico de Monte Verde (región de Los Lagos).

1 J.M. Perceval 10259, Vitacura, Santiago, Chile.

$\measuredangle$ juanfranciscocampodonico@gmail.com

2 Entomology Department, School of Natural Resource Sciences, North Dakota State University, Dept. 7650, P.O. Box 6050; Fargo, ND, USA.

ed.faundez@gmail.com

3 Geosciences Department, North Dakota State University, Dept. 6050, P.O. Box 6050; Fargo, ND, USA.

4 Departamento de Zoología Médica, Centro de Estudios en Biodiversidad (CEBCh), Magallanes 1979, Osorno, Chile. 


\section{MATERIALES Y MÉTODOS}

La fotografía fue tomada con una cámara digital adaptada a un microscopio estereoscópico. El material examinado se encuentra depositado en el Pabellón de Colecciones Biológicas "Prof. Edmundo Pisano", Instituto de la Patagonia, Universidad de Magallanes, Chile (IDEP). Todas las medidas se encuentran en milimetros. En sistemática y terminología se sigue a Szwedo (2004a). La edad del fósil fue datada en base a radiocarbono (Hoganson et al. 1989; Ashworth \& Hoganson, 1993) y corregido para los efectos del yacimiento de acuerdo a Reimer et al. (2013).

\section{RESULTADOS}

Mapuchea pinoi sp. nov. (Figs. 1 y 2)

Descripción: Tegmen (Figs. 1 y 2) de largo máximo 3,835 mm, ancho máximo $1,994 \mathrm{~mm}$, puntuación marcada, forma irregularmente oval, subrectangular en la base, margen costal no engrosado, curvatura leve, pronunciándose en el tercio apical; venas $\mathrm{Cu}, \mathrm{M}$ y $\mathrm{R}$ fusionadas en la porción basal, formando antes de su separación en el primer tercio del tegmen una franja conspicua de superficie lisa que contrasta respecto al resto de la superficie del tegmen; vena radial con dos tubérculos ovales separados por $0,457 \mathrm{~mm}$, el anterior $(0,27 \mathrm{~mm}$ de largo $)$ ubicado antes de la mitad del largo del tegmen, de poco más de dos tercios del largo del posterior $(0,375 \mathrm{~mm}$ de largo); vena radial formando una celda con la vena medial; vena medial con dos tubérculos ovales separados por 0,482 mm, ambos en una posición ligeramente más basal en relación a los tubérculos primero y segundo de la vena radial, respectivamente, el primero $(0,171 \mathrm{~mm}$ de largo $)$ poco más de la mitad del largo del segundo $(0,301$ $\mathrm{mm}$ de largo); vena cubital con un prominente tubérculo triangular $(0,813 \mathrm{~mm}$ de largo $)$ que ocupa alrededor de un tercio de la extensión de esta, ubicado hacia la mitad del largo del tegmen, sutura claval con una cresta sigmoidea, de 1,295 mm de largo, la cual comienza cerca de la base del tegmen y se extiende hasta más de un tercio del largo de este, ocupando alrededor de dos tercios de la extensión de la sutura claval, la cresta es más prominente hacia los extremos respecto a su porción media y se encuentra rematada por una franja de superficie lisa, la cual presenta una pequeña rama dirigida basalmente en la mitad; vena claval con una cresta de 0,336 $\mathrm{mm}$ de largo ubicada en su porción media, más cercana a la base.
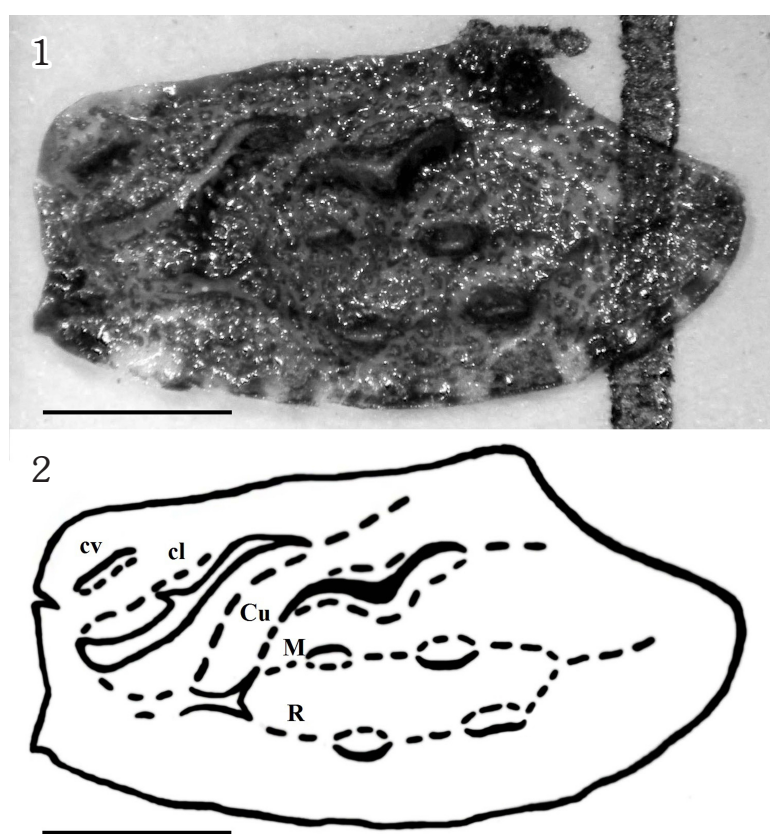

Figs. 1 y 2. Mapuchea pinoi sp. nov., tegmen izquierdo, escala: $1 \mathrm{~mm}$. 1. Fotografía; 2. Dibujo. cv vena claval, cl sutura claval, $\mathrm{Cu}$ vena cubital, $\mathrm{M}$ vena medial, $\mathrm{R}$ vena radial.

Holotipo: Chile, región de Los Lagos, Monte Verde, orillas de la cala de Chinchihuapi, lat. $-41,503365^{\circ}$, long. $-73,207750^{\circ}, 12 \mathrm{~km}$ en dirección sudoeste del edificio terminal del aeropuerto de El Tepual. Extraído de la base de un lecho de turba que Mario Pino (Universidad Austral de Chile, Valdivia) nombró la unidad de Monte Verde [IDEP]. La edad de radiocarbono para el horizonte es $11.600 \pm 120$ años AP (TX-3472), lo que calibrado se traduce en una probabilidad mediana para esta fecha de 13.429 cal años A.P. Ejemplar montado en placa, ubicado en posición número 1.

Etimología: Esta especie está dedicada a Mario Pino, quien aportó una interpretación sedimentológica y un marco estratigráfico para acompañar los importantes hallazgos 
arqueológicos de Monte Verde (ver Dillehay, 1989).

\section{DISCUSIÓN}

Mapuchea pinoi sp. nov. se diferencia de las demás especies del género por presentar la sutura claval con una larga y conspicua cresta en el tercio basal del tegmen (dos crestas claramente diferenciadas en la sutura claval de $M$. hamiltoni; un tubérculo inconspicuo en la base y una cresta conspicua en la mitad apical de la sutura claval en $M$. chilensis; un tubérculo conspicuo en la base y tres tubérculos inconspicuos a lo largo de la sutura claval en $M$. burckhardti).

El estudio de los insectos fósiles de Monte Verde forman parte un proyecto que incluye insectos fósiles de la región de Los Lagos (en sentido antiguo, considerando la actual región de los Ríos), desde el último máximo glacial hasta el Holoceno (Ashworth \& Hoganson, 1993). En ese trabajo se demostró que desde los 18.000 a los 14.000 años AP, la vegetación de la región de Los Lagos era mayoritariamente páramo. La fauna de coleópteros de tierras bajas consistía de unas pocas especies, dominado por taxones que hoy en día se encuentran en las partes altas de los Andes y zonas costeras. Hace 14.000 años, el páramo fue reemplazado por diversos tipos de bosques, los que dominaron las tierras bajas hasta la deforestación realizada para la agricultura a mediados del siglo XIX (Ashworth \& Hoganson, 1993; Hoganson et al. 1989). De este modo, el cambio en la biota de hace 14.000 años fue causado por un cambio climático rápido en un rango de $4-5^{\circ} \mathrm{C}$. Los cazadores-recolectores humanos aparecieron hacia el final de la transición a un clima más cálido (Ashworth \& Hoganson, 1993).

En este escenario, el lecho de turba de la unidad de Monte Verde selló y ayudó a preservar los importantes artefactos prehistóricos. El fósil de Mapuchea pinoi sp. nov. fue enumerado como un hemíptero en Hoganson et al. (1989); en donde los fósiles de insectos del sitio se consideran contemporáneos a la ocupación humana. En esta zona se han recolectado otros hemípteros modernos considerados relictos; tales como Archaeoditmotarsus crassytilus
Faúndez, Carvajal \& Rider, 2014 y Nerthra (Rhinodermacoris) praecipua Todd, 1957 (Faúndez et al. 2014; Faúndez \& Ashworth, 2015). Por esta razón creemos posible que $M$. pinoi se encuentre presente actualmente y no ha sido recientemente colectada.

\section{LITERATURA CITADA}

Ashworth, A. C., \& Hoganson, J. W. (1993). The magnitude and rapidity of the climate change marking the end of the Pleistocene in the mid-latitudes of South America. Palaeogeography, Palaeoclimatology, Palaeecology, 101, 263-270.

Dillehay. T. D. (Ed.) (1989). Monte Verde-A Late Pleistocene Settlement In Southern Chile. Washington D.C.: Smithsonian Institution Press.

Faúndez, E. I., Carvajal, M. A., \& Rider, D. A. (2014). Archaeoditomotarsus crassitylus, gen. and sp. nov. from Chile. Zootaxa, 3860(1), 87-9.

Faúndez, E. I., \& Ashworth, A. C. (2015). Notas sobre la familia Gelastocoridae (Hemiptera: Heteroptera) en el extremo sur de Chile, con descripción de un subgénero y especie nuevos. Anales del Instituto de la Patagonia, 43(2), 69-74.

Hoganson, J. W., Gunderson, M. J., \& Ashworth, A. C. (1989). Fossil Beetle Analysis. En T. D. Dillehay (Ed.), Monte Verde-A Late Pleistocene Settlement In Southern Chile (pp. 211-226). Washington D.C.: Smithsonian Institution Press.

Nielson, M. W. (1996). A new species of Myerslopia from Chile (Homoptera: Cicadellidae). Entomological News, 107(5), 322-326.

Rakitov, R. (2015). Observations on the biology and anatomy of Myerslopiidae (Hemiptera, Membracoidea). Psyche: A Journal of Entomology, 10 pp.

Reimer, P. J., Bard, E., Bayliss, A., Beck, J. W., Blackwell, P. G., Bronk Ramsey, C., Buck, C. E., .. \& van der Plicht, J. (2013). IntCal13 and MARINE13 radiocarbon age calibration curves 0-50000 years calBP. Radiocarbon, 55(4), 1869-1887.

Szwedo, J. (2004a). A new genus and six new 
species of ground-dwelling leafhoppers from Chile and New Zealand (Hemiptera: Cicadomorpha: Myerslopiidae). Zootaxa, 424, 1-20.
Szwedo, J. (2004b). An annotated checklist of Myerslopiidae with notes on the distribution and origin of the group (Hemiptera: Cicadomorpha). Zootaxa, 425, 1-15. 